\title{
Intentions and Factors Regarding Selection of Workplace by Postgraduate Dental Trainees after Clinical Training
}

\author{
Yoshikazu Okawa ${ }^{1)}$, SoIchiro Hirata ${ }^{2)}$, Ikuo Nasu ${ }^{3)}$ and Yukio Hirata ${ }^{4)}$ \\ ${ }^{1)}$ Department of Dental Hygiene, Faculty of Health Care Science, \\ Chiba Prefectural University of Health Sciences, \\ 2-10-1 Wakaba, Mihama-ku, Chiba 261-0014, Japan \\ 2) Department of Social Dentistry, Tokyo Dental College, \\ 2-9-18 Misaki-cho, Chiyoda-ku, Tokyo 101-0061, Japan \\ ${ }^{3)}$ Department of Dental Public Health, Nihon University School of Dentistry at Matsudo, \\ 2-870-1 Sakaechonishi, Matsudo, Chiba 271-8587, Japan \\ 4) Department of Dental Sociology, Graduate School of Dentistry, \\ Kanagawa Dental University, \\ 82 Inaoka-cho, Yokosuka, Kanagawa 238-8580, Japan
}

Received 11 April, 2016/Accepted for publication 28 June, 2016

\begin{abstract}
The purpose of the present study was to investigate the reasons governing selection of geographical location of workplace by postgraduate dental trainees. We believe that such data would aid in the development of measures aimed at reducing regional disparities in the number of dentists. A total of 10 dental colleges and faculties in Japan were invited to participate in the study. Anonymous, self-completed questionnaire surveys were given to 739 dentists at 13 clinical training facilities following training in 2012 and 2013; 713 responses were analyzed. The items surveyed included the local population in the area in which they wished to work and whether they wanted to work in rural areas or remote islands. The most common response to the question regarding population was "medium-size cities" with a population of 100,000 to 300,000 people (67.3\%). The largest number of respondents stated that they hoped to work in their hometown or a neighboring area (49.9\%). A large percentage wished to work in medium-size cities, which may reflect the population of their hometowns. The current system allows dentists a free choice in becoming practitioners or hospital dentists on completion of training. This may explain why so many expressed a preference to work in their hometown or a neighboring area here. These results suggest that policies relying on increasing the supply of dentists as the sole solution to rural shortages are flawed.
\end{abstract}

Key words: Dentists - Population — Health services accessibility — Geographic locations - Postgraduate dental trainees 


\section{Introduction}

One important task under national health care policy is to improve dental care by determining its accessibility and thereby identifying where improvements are needed. Both the number of dental schools and their admission capacity saw an increase between 1970 and 1980 in Japan. As a result, the total number of dentists exceeded 100,000 in 2010, reaching 102,551 (80 dentists per 100,000 population) by $2014^{6)}$, suggesting that supply may outstrip demand in the future. Although regional disparities in dental care services are gradually being alleviated as the total number of dentists increases ${ }^{11,14)}$, a shortage of dentists still persists in rural and depopulated areas ${ }^{11)}$. One survey by the Ministry of Health, Labour, and Welfare of Japan in 2009 attempted to ascertain the number of areas with no accessibility to medical or dental services. The results revealed that there were 930 areas where there was no access to a dentist (total population in these areas: 236,527), which represented a population even greater than that with no access to a physician (total population in such areas: 136,272) ${ }^{7}$. "Areas without physicians/dentists" were defined as local areas in which 50 or more residents lived within a 4-kilometer radius of a central area but did not have easy access to health care institutions, including dental clinics. Although another analysis of the geographic distribution of dental clinics in Japan suggested that regional disparities were alleviated between 2000 and 2010, there was no dental clinic in $21 \%$ of municipalities with a population of less than 5,000, and this percentage has largely remained unchanged since $2000^{12}$.

When developing measures aimed at alleviating such regional disparities in the number of dentists and dental care services, it is important to take the intentions of dental school graduates into consideration, especially those pertaining to career path and preferred geographical location of workplace. In Japan, dental school graduates who have also passed the national examination have traditionally been eligible for a license to practice dentistry. In 2006, however, a new system was introduced that required such newly qualified dentists to undergo further clinical training for at least 1 year at this point before embarking on a career. This was because, under the old system, as students, they would not have been permitted to engage in actual clinical practice during their time at dental school, so they would have had no practical experience on graduating university $^{4}$. Therefore, this new postgraduate education system enables freshly qualified dentists to undergo clinical training under professional supervision so that they may gain real clinical experience before commencing such work under their own responsibility. Patient needs continue to diversify, partially as a result of the aging of the Japanese population, and partly in response to the increasing sophistication and specialization of dental techniques. In this context, the clinical training system aims to train dentists who can respond to the aging society and declining birth rate in the near future, as well as to changes in the structures of diseases.

More than $80 \%$ of applicants for such training are presently accepted by hospitals affiliated with dental schools. As a result, the number of trainee dentists in prefectures with dental college-affiliated hospitals is significantly larger than in those without, further exacerbating regional disparities ${ }^{2}$. Shortages of dentists are particularly pronounced in mountainous and other depopulated areas, including remote islands. In such areas, geographical remoteness can lead to delays in identifying and treating dental disorders, which can worsen the severity of a disease. Therefore, a system needs to be established that would enable local residents in such areas to receive the dental care and health services they require close to home. The purpose of the present study was to survey students undergoing postgraduate clinical training in order to investigate factors influencing their future selection of geographical location of place of work. We believe that this data will provide a useful tool in developing policy aimed at alleviating regional disparities in the 
number of dentists.

\section{Materials and Methods}

There are 29 dental schools in Japan, located in 19 out of a total of 47 prefectures. A total of 10 dental schools located in 9 prefectures (Hokkaido, Niigata, Tokyo, Chiba, Aichi, Osaka, Okayama, Fukuoka, and Kagoshima) were invited to participate in the survey. Clinical training must be conducted in hospitals affiliated with colleges or universities with dental or medical courses (excluding those that do not provide dental care services) or hospitals or clinics designated by the Ministry of Health, Labour, and Welfare. In March 2012 and 2013, anonymous, self-completed surveys were given to 739 postgraduate dental trainees at 13 institutions, including university hospitals, on completion of clinical training. A total of 713 responses (valid response rate, $96.5 \%$ ) were analyzed. The total number of respondents comprised 418 men (58.6\%) and 295 women $(41.4 \%)$ with a mean age of 27.2 years (27.7 and 26.5 years for men and women, respectively).

The items surveyed included sex, age, intended future career (after completing postgraduate clinical training), population of the area in which they wished to work as hospital dentists or practitioners following postgraduate clinical training, and whether they wanted to work in rural and depopulated areas. In response to items regarding "Working in depopulated areas as their future career" and "Population of the area in which they want to work in the future", the participants were asked to choose from the following 5 options: (1) "strongly hope"; (2) "hope"; (3) "undecided"; (4) "do not hope"; and (5) "do not hope at all".

The odds ratios (ORs) and 95\% confidence intervals (CIs) were determined using stepwise logistic regression analysis for the 507 respondents who answered all survey items. The dependent variable was set at 1 if they wanted to work in remote areas, and at 0 if they did not. Other survey items (or factors) as independent variables were set at 1 if they answered the question as "significantly hope" or "hope", and at 0 if they did not. The dummy variable was set at 1 if the age of the postgraduate dental trainee was 30 years or more, and at 0 if it was less than 30 years. All statistical analyses were performed with EZR (Saitama Medical Center, Jichi Medical University, Saitama, Japan), a graphical user interface for R (The R Foundation for Statistical Computing, Vienna, Austria) $)^{3)}$. More precisely, it is a modified version of $\mathrm{R}$ commander, and is designed to add statistical functions frequently used in biostatistics.

\section{Results}

\section{Future career of postgraduate dental trainees}

To the item: "Future career after completing postgraduate clinical training", $77.8 \%$ of the respondents answered "I strongly hope/ hope to work in dental clinics to start my career". Of these, $59.5 \%$ wished to become certified specialists, and $56.8 \%$ hoped to work as practitioners in the future. A total of $25.7 \%$ hoped to continuously work as hospital dentists (Table 1).

\section{Population of area in which postgraduate dental trainees want to work}

Regarding population, the largest number of postgraduate dental trainees (67.3\%) wished to work as hospital dentists or practitioners in medium-sized cities with a population of 100,000 to 300,000 ; the smallest number of respondents $(20.1 \%)$ preferred towns and villages with a population of less than 30,000 (Table 2).

\section{Reasons for selection of place of work as hospital dentists or practitioners in relation to population}

The largest number of postgraduate dental trainees $(49.9 \%)$ chose a particular geographical location as "It was their hometown or its surrounding area"; the second largest number $(17.7 \%)$ cited "The convenience of the 
Table 1 Future career of postgraduate dental trainees (multiple answers allowed)

\begin{tabular}{|c|c|c|c|c|c|c|}
\hline \multirow[b]{2}{*}{ Future careers } & \multicolumn{2}{|c|}{ Strongly hope } & \multicolumn{2}{|l|}{ Hope } & \multicolumn{2}{|l|}{ Total } \\
\hline & $\begin{array}{l}\text { Number of } \\
\text { respondents }\end{array}$ & $\%$ & $\begin{array}{l}\text { Number of } \\
\text { respondents }\end{array}$ & $\%$ & $\begin{array}{l}\text { Number of } \\
\text { respondents }\end{array}$ & $\%$ \\
\hline Work in dental clinics (to start their careers) & 195 & 27.3 & 360 & 50.5 & 555 & 77.8 \\
\hline $\begin{array}{l}\text { Acquisition of certificates as specialized and } \\
\text { certified dentists }\end{array}$ & 188 & 26.4 & 236 & 33.1 & 424 & 59.5 \\
\hline Practitioners in the future & 231 & 32.4 & 174 & 24.4 & 405 & 56.8 \\
\hline Involvement in community-based medicine & 78 & 10.9 & 229 & 32.1 & 307 & 43.1 \\
\hline $\begin{array}{l}\text { Work for health care institutions other than } \\
\text { university hospitals }\end{array}$ & 41 & 5.8 & 238 & 33.4 & 279 & 39.1 \\
\hline Overseas study & 76 & 10.7 & 194 & 27.2 & 270 & 37.9 \\
\hline Acquisition of degrees & 128 & 18.0 & 114 & 16.0 & 242 & 33.9 \\
\hline $\begin{array}{l}\text { University hospital from which the respond- } \\
\text { ents graduated }\end{array}$ & 66 & 9.3 & 165 & 23.1 & 231 & 32.4 \\
\hline Advancement to graduate school & 111 & 15.6 & 104 & 14.6 & 215 & 30.2 \\
\hline $\begin{array}{l}\text { Hoping to work continuously as hospital den- } \\
\text { tists }\end{array}$ & 32 & 4.5 & 151 & 21.2 & 183 & 25.7 \\
\hline $\begin{array}{l}\text { Work in the field of international health coop- } \\
\text { eration }\end{array}$ & 34 & 4.8 & 145 & 20.3 & 179 & 25.1 \\
\hline Work for other university hospitals & 22 & 3.1 & 85 & 11.9 & 107 & 15.0 \\
\hline $\begin{array}{l}\text { Work in administrative agencies (including } \\
\text { prefectural health centers) }\end{array}$ & 12 & 1.7 & 76 & 10.7 & 88 & 12.3 \\
\hline $\begin{array}{l}\text { Non-clinical (basic) researchers in universities } \\
\text { and institutions }\end{array}$ & 13 & 1.8 & 43 & 6.0 & 56 & 7.9 \\
\hline
\end{tabular}

Table 2 Population of area in which postgraduate dental trainees want to work as hospital dentists or practitioners*

\begin{tabular}{|c|c|c|c|c|c|c|}
\hline \multirow[b]{2}{*}{ Population } & \multicolumn{2}{|c|}{ Strongly hope } & \multicolumn{2}{|l|}{ Hope } & \multicolumn{2}{|l|}{ Total } \\
\hline & $\begin{array}{l}\text { Number of } \\
\text { respondents }\end{array}$ & $\%$ & $\begin{array}{l}\text { Number of } \\
\text { respondents }\end{array}$ & $\%$ & $\begin{array}{l}\text { Number of } \\
\text { respondents }\end{array}$ & $\%$ \\
\hline $\begin{array}{l}\text { Large cities } \\
\text { (with a population of more than } 500,000 \text { ) }\end{array}$ & 110 & 15.4 & 212 & 29.7 & 322 & 45.2 \\
\hline $\begin{array}{l}\text { Medium-sized cities } \\
\text { (with a population of } 100,000 \text { to } 300,000 \text { ) }\end{array}$ & 100 & 14.0 & 380 & 53.3 & 480 & 67.3 \\
\hline $\begin{array}{l}\text { Cities } \\
\text { (with a population of } 50,000 \text { to } 100,000 \text { ) }\end{array}$ & 95 & 13.3 & 347 & 48.7 & 442 & 62.0 \\
\hline $\begin{array}{l}\text { Towns and villages } \\
\text { (with a population of } 30,000 \text { to } 50,000 \text { ) }\end{array}$ & 39 & 5.5 & 187 & 26.2 & 226 & 31.7 \\
\hline $\begin{array}{l}\text { Towns and villages } \\
\text { (with a population of less than } 30,000 \text { ) }\end{array}$ & 29 & 4.1 & 114 & 16.0 & 143 & 20.1 \\
\hline
\end{tabular}

*Respondents who "strongly hope" or "hope" to work in these areas (multiple answers allowed) $\quad(\mathrm{n}=713)$ 
Table 3 Reasons for selection of place of work as hospital dentists or practitioners in relation to population

\begin{tabular}{lcr}
\hline \multicolumn{1}{c}{ Reasons for the selection } & $\begin{array}{c}\text { Number of } \\
\text { respondents }\end{array}$ & $\%$ \\
\hline I come from the area or its surrounding areas & 356 & 49.9 \\
The area is convenient to live & 126 & 17.7 \\
The population is large & 61 & 8.6 \\
The area is a large city (e.g., Tokyo and others) & 48 & 6.7 \\
There is a shortage of dentists in the area & 21 & 2.9 \\
The university from which I graduated is in the area & 13 & 1.8 \\
Other reasons & 6 & 0.8 \\
No response & 82 & 11.5 \\
\hline
\end{tabular}

Table 4 Whether postgraduate dental trainees want to work in rural areas or remote islands

\begin{tabular}{lcr}
\hline \hline & $\begin{array}{c}\text { Number of } \\
\text { respondents }\end{array}$ & $\%$ \\
\hline I am very interested in working in these areas & 31 & 4.3 \\
I will work in these areas depending on conditions & 304 & 42.6 \\
I do not want to work in these areas & 224 & 31.4 \\
I can not decide & 153 & 21.5 \\
No response & 1 & 0.1 \\
\hline & & $(\mathrm{n}=713)$
\end{tabular}

area" as the reason (Table 3).

\section{Whether postgraduate dental trainees want to work in rural areas or remote islands}

A total of 304 respondents $(42.6 \%)$ stated that they could work in rural areas or remote islands depending on the conditions; 31 $(4.3 \%)$ wished to work in those areas (Table 4).

\section{Specific conditions required by} postgraduate dental trainees who stated that "they can work in rural areas or remote islands depending on the conditions"

Regarding the work conditions required by the 304 postgraduate dental trainees, the larg- est number of respondents (50.3\%) hoped to receive "Financial support provided by the central and local governments". This was followed by "As personnel dispatched by university hospitals and other institutions" (14.8\%); "Development of dental clinics in depopulated areas by local governments" (14.5\%); and "Technical support provided by specialists and the establishment of training systems" (14.1\%) (Table 5).

\section{Factors related to whether postgraduate dental trainees want to work in rural areas}

The ORs of factors related to whether postgraduate dental trainees wanted to work in rural areas and the 95\% CIs were calculated. The ORs of the following items were significant: "Advancement to graduate school" 
Table 5 Specific conditions required by postgraduate dental trainees who stated that "they can work in rural areas or remote islands depending on conditions" $(\mathrm{n}=304)$

\begin{tabular}{lrr}
\hline \hline & $\begin{array}{c}\text { Number of } \\
\text { respondents }\end{array}$ & $\%$ \\
\hline Financial support provided by the central and local governments & 153 & 50.3 \\
As personnel dispatched by university hospitals and other institutions & 45 & 14.8 \\
Development of dental clinics in depopulated areas by local governments & 44 & 14.5 \\
Technical support provided by specialists and the establishment of training systems & 43 & 14.1 \\
Securing the appropriate number of dental hygienists and technicians & 11 & 3.6 \\
Other responses & 7 & 2.3 \\
No response & 1 & 0.3 \\
\hline
\end{tabular}

Table 6 Odds ratio of factors related to whether postgraduate dental trainees want to work in rural areas and $95 \%$ confidence interval $(\mathrm{n}=507)$ *

\begin{tabular}{lcc}
\hline \hline \multicolumn{1}{c}{ Factor } & OR (95\%CI) & p-value \\
\hline Advancement to graduate school & $1.77(1.25-2.50)$ & 0.001 \\
Work in the field of international health cooperation & $1.95(1.35-2.82)$ & 0.000 \\
Towns and villages (with a population of 30,000 to 50,000) & $1.89(1.23-2.88)$ & 0.003 \\
Towns and villages (with a population of less than 30,000) & $2.47(1.47-4.15)$ & 0.001 \\
\hline
\end{tabular}

*507 respondents answered all survey items

(OR: 1.77; 95\%CI: 1.25-2.50); "Work in the field of international health cooperation" (OR: 1.95; 95\%CI: 1.35-2.82); "Towns and villages (with a population of 30,000 to 50,000$)$ " (OR: 1.89; 95\%CI: 1.23-2.88); and "Towns and villages (with a population of less than 30,000)" (OR: 2.47; 95\%CI: 1.47-4.15) (Table $6)$.

\section{Discussion}

The present results revealed that a large percentage of postgraduate dental trainees hoped to work in areas with a population of 50,000 to 300,000. An American survey suggested that only $7.65 \%$ of professionally active dentists in the U.S. work in areas with a population of less than $50,000^{5}$. Taken together, this suggests that selection of a place to work is related to the populations of the municipalities of their hometowns. The Japanese health care system allows dental students to freely choose where they work on completion of training, whether in private practice or a hospital setting. This may also explain why many prefer to return to their hometown or a nearby area to begin their career, as has been suggested by the results of a survey involving medical students and postgraduate medical trainees ${ }^{13)}$. Similar findings have been reported in other countries: the results of American surveys showed that dental students in the U.S. also wished to work in the areas in which they were born and raised ${ }^{1}$. However, the proportion of dentists is very high in major cities with a population of over 500,000 in Japan. This makes competition to provide better dental care services very severe, forcing many dental clinics into financial difficulties. This may explain why postgraduate dental trainees in the present study preferred areas with a population of only 50,000 to 300,000 .

On the other hand, where the population 
concerned is smaller than this, municipalities may find it difficult to continue to secure an adequate number of dentists after clinical training, even though policy may be designed to encourage this. Studies have yet to determine the right level for the spatial competition hypothesis suggested by one economic model $^{9,10)}$, which stipulates that an increase in the total number of physicians will alleviate disparities in their geographic distribution. This should be equally applicable to dentists. In fact, due to the increase in the number of dentists over twenty years from 1980 to 2000 , practicing dentists have spread more evenly ${ }^{11)}$. However, such a reduction in disparity of geographic distribution remains to be seen. One possible solution might be to entice dentists to work in towns and villages with a small population of less than 30,000 by a range of incentives such as preferential tax treatment and special reimbursements for treatments provided in specific areas under the health insurance system.

In the present study, $42.6 \%$ of postgraduate dental trainees stated that they would be willing to work in rural areas or remote islands depending on the conditions, while $4.3 \%$ said they wished to work in such areas. This means that a total of $47 \%$ are interested in working in mountainous and other depopulated areas, including remote islands, and contributing to community-based medicine. We believe that many such trainees will also have demonstrated an interest in international cooperation on healthcare, suggesting the desire to bring dental health care to sparsely populated areas as a strong motivation in such cases. Moreover, $50.3 \%$ stated that they would work in rural and depopulated areas as long as they could receive "financial support provided by the central and local governments" as they would be required to implement dental care services on a small budget. It is difficult to operate dental clinics in depopulated areas, and this issue needs to be addressed. The Japanese (universal) health insurance system stipulates the reimbursement of medical fees for specific treatment procedures to be paid based on the national standard, which means that it is necessary to provide care for at least a specific minimum number of patients to operate a dental clinic. The second largest number of respondents stated that "they can work in rural and depopulated areas as dentists dispatched by university or other hospitals". This may be because they believe that they would be able to return to those hospitals after serving such a community, ensuring a specific income at some later date. Although some local governments allocate subsidies to dental clinics in rural areas with few dentists and invite postgraduate dental trainees to work there, it is still difficult to alleviate regional disparities and secure appropriate numbers of dentists. Among respondents to this survey, $31.4 \%$ stated that they did not want to work in rural areas or remote islands. Their reasons were as follows: "I want to work in my hometown or its surrounding area" and "Rural areas are inconvenient places to live". These respondents presumably chose their intended place of work based on geographical convenience and the educational environment available for their children. Some respondents said they wished to work in urban areas so that they could encounter a larger number of clinical cases and gain more experience of using advanced dental care techniques, which would help them develop their careers.

The above responses suggest that it might be effective to introduce a system of certification of specialization as a way of motivating urban dentists to work in remote areas with little competition, as this would help them with their careers. Other such policies might include the expansion and area-targeting of enrollment systems in dental schools; scholarships for dentists who have worked in remote areas for a specific period of time; and conducting training and workshops in remote areas. Active encouragement of women taking on an increasing role in the workplace has seen an increase in the number of women being admitted to dental schools over recent years, and as of 2014 women have occupied more than $40 \%$ places on training programs ${ }^{8)}$. To improve community-based dental care, it 
is also necessary to develop a social environment conductive to helping female dentists maintain a balance between their dental practice and private life, including marriage, childbirth, and child-raising.

The health care institutions participating in the present study were selected taking geographic location into consideration. A complete survey involving dentists in clinical training at all 29 colleges in Japan was not conducted, and this may have distorted some of the results. Further larger scale and longitudinal surveys are needed to determine which factors influence the selection of place of work by dentists and the status of regional disparities in more detail.

\section{References}

1) Graham JW (1977) Factors influencing the choice of practice location for recent dental graduates. J Am Dent Assoc 94:821-825.

2) Hirata $S$, Mataki $S$, Akiyama $H$, Nitta $H$, Okada M, Sakayori T, Sugito H, Ishii T (2009) Geographic distribution of postgraduate dental trainee in Japan. Bull Tokyo Dent Coll 50: 63-70.

3) Kanda Y (2013) Investigation of the freely available easy-to-use software 'EZR' for medical statistics. Bone Marrow Transplant 48: 452-458.

4) Kikuchi M (2007) The Japanese system of dental clinical training. Prosthodont Res Pract 6: 78-80.

5) McFarland KK, Reinhardt JW, Yaseen M (2010) Rural dentists of the future: dental school enrollment strategies. J Dent Educ 74: 830-835.

6) Ministry of Health, Labour, and Welfare (2015) Annual Health, Labour and Welfare Report 2013-2014. At: http://www.mhlw.go. jp/english/wp/wp-hw8/dl/02e.pdf (Accessed: December 23, 2015)
7) Ministry of Health, Labour, and Welfare (2015) An overview of 2009 surveys of areas without physicians and dentists. At: http://www.mhlw.go.jp/stf/houdou/ 2r9852000000ulrk-img/2r9852000000ult1. pdf (Accessed: December 23, 2015) (in Japanese)

8) Ministry of Health, Labour, and Welfare (2014) An overview of Survey of Physicians, Dentists and Pharmacists 2014. At: http:// www.mhlw.go.jp/toukei/saikin/hw/ishi/14/ (Accessed: December 23, 2015) (in Japanese)

9) Newhouse JP (1990) Geographic access to physicians services. Annu Rev Public Health 11:207-230

10) Newhouse JP, Williams AP, Bennett BW, Schwartz WB (1980) Where have all doctors gone? JAMA 247:2392-2396.

11) Okawa Y, Hirata S, Okada M, Ishii T (2011) Geographic distribution of dentists in Japan. J Public Health Dent 71:236-240.

12) Okawa Y, Hirata $S$ (2014) Trends in the geographic distribution of dental clinics in Japan. Community Dent Health 31:62-64.

13) Takeda Y, Otaki J, Takahashi M, Morio K, Takada M, Inafuku T, Yasui H, Takaya A, Kai I (2010) A survey of background factors influencing disparities in the distribution of physicians (the first report), The status of the selection of careers by medical students and interns, and factors influencing the selection of the clinical department and place of works. Jpn Med J (Natl Inst Health Jpn) 4471:101-107. (in Japanese)

14) Toyokawa S, Kobayashi Y (2010) Increasing supply of dentists induces their geographic diffusion in contrast with physicians in Japan. Soc Sci Med 71:2014-2019.

\section{Correspondence:}

Dr. Yoshikazu Okawa

Department of Dental Hygiene,

Faculty of Health Care Science,

Chiba Prefectural University of Health

Sciences,

2-10-1 Wakaba, Mihama-ku,

Chiba 261-0014, Japan

E-mail: yoshikazu.okawa@cpuhs.ac.jp 